\title{
Preparando a las nuevas generaciones con un enfoque organizacional tipo Hollywood a través del aprendizaje basado en Proyectos colaborativos
}

\section{Preparing new generations with a Hollywood-type organizational approach through collaborative project-based learning}

\author{
DOI: $10.46932 / \mathrm{sfjdv2n5-029}$
}

Received in: Jun 1st, 2021

Accepted in: Sep 30th, 2021

\begin{abstract}
M. en C. Eva Valdez Alemán
Docente de la División de Electrónica del Tecnológico de Estudios Superiores de Ecatepec; Av. Tecnológico S/N C.P. 55210 Col. Valle de Anáhuac, Ecatepec de Morelos, Estado de México Correo electrónico: evaldez@ tese.edu.mx

Mercedes Flores Flores

Docente de la División de Electrónica del Tecnológico de Estudios Superiores de Ecatepec; Av. Tecnológico S/N C.P. 55210 Col. Valle de Anáhuac, Ecatepec de Morelos, Estado de México Correo electrónico: mflores@ tese.edu.mx

\section{Griselda Cortés Barrera}

Docente de la División de Electrónica del Tecnológico de Estudios Superiores de Ecatepec; Av. Tecnológico S/N C.P. 55210 Col. Valle de Anáhuac, Ecatepec de Morelos, Estado de México

Correo electrónico: gcortes@ tese.edu.mx
\end{abstract}

\section{RESUMEN}

El objetivo principal de esta investigación es lograr que, con el aprendizaje basado en proyectos colaborativos, los alumnos adquieran un enfoque organizacional tipo Hollywood.

Esta investigación es de corte cuantitativo; se realizó la recolección de datos con el fin de explicar las causas que implicarán en el alumno de pasar de un aprendizaje basado en proyectos colaborativos hasta llegar a desarrollar un proyecto desde el punto de vista organizacional tipo Hollywood (causa-efecto). Al finalizar dicha investigación se observó que los alumnos se sienten preparados para desempeñarse en esta modalidad; que el aprendizaje basado en proyectos colaborativos, sí propicia a trabajar en la modalidad organizacional tipo Hollywood y también considerando que han trabajado semestre con semestre proyectos diferentes estarían dispuestos a realizarlos para problemáticas reales.

Palabras Clave: Aprendizaje Basado en Proyectos, Aprendizaje Colaborativo, Organización tipo Hollywood.

\section{ABSTRACT}

The main objective of this research is to make the learning based on collaborative projects, pupils a Hollywood type organizational approach.

This research is quantitative nature; Data collection was performed in order to explain the causes that involve the student to move from a system based on collaborative learning projects up to develop a project from an organizational point of view Hollywood type (cause and effect).

Upon completion of this investigation it was found that students feel prepared to work in this mode; learning based on collaborative projects if conducive to work in Hollywood kind of organizational 
modality and also considering that half have worked with half different projects would be willing to do them for real problems.

Keywords: Project Based Learning, Collaborative Learning, Hollywood type organization.

\section{INTRODUCCIÓN}

Las nuevas generaciones cada vez requieren nuevos enfoques para el buen desempeño en su desarrollo académico y posteriormente en su campo laboral; cabe señalar que nos referimos en esencia a aquellas generaciones que están por egresar de un nivel superior.

Si bien mantener a los alumnos motivados y comprometidos con el estudio es todo un reto, la inclusión de tener una alternativa laboral al egresar se convierte en otro reto al lograr transmitirles esta nueva modalidad y de obtener resultados positivos.

Partiendo que una de las principales características de la Reforma para la Educación, es la formación por competencias, la cual pretende que los individuos se formen de una manera eficiente bajo su contexto; lo que permite generar aprendizajes significativos y formen parte de los conocimientos que preparará al alumno para una vida laboral.

Aprovechando la forma de trabajar con el aprendizaje basado en proyectos puede enlazar a la idea de una empresa que se monta para hacer un proyecto, y que posteriormente se desmonta. Es decir agencias que construyen "paquetes de personas profesionales" que pueden llevar a cabo el trabajo requerido. Como en la forma actual de reproducir películas de Hollywood.

El objetivo principal de esta investigación es lograr que, con el aprendizaje basado en proyectos colaborativos, los alumnos adquieran un enfoque organizacional tipo Hollywood.

\section{FUNDAMENTOS TEÓRICOS}

El Aprendizaje Basado en Proyectos (ABP) es esencialmente un sistema de aprendizaje estratégico que combina distintos principios educativos complementarios para mejora del proceso instructivo. Esta especialmente dirigido a potenciar y optimizar un proceso de aprendizaje centrado en el alumno, en contextos colaborativos, integrado, auto dirigido y reflexivo. M.C.E. Gwee Kaohsiguing J.M. Sci. (2009)

Este modelo tiene sus raíces en el constructivismo, que evoluciono a partir de los trabajos de psicólogos y educadores tales como Lev Vygostsky, Jerome Bruner, Jean Piaget y John Dewey. El constructivismo se apoya en la creciente comprensión del funcionamiento del cerebro humano, en cómo almacena y recupera información, cómo aprende y cómo el aprendizaje acrecienta y amplía el aprendizaje previo. 
El constructivismo enfoca al aprendizaje como el resultado de construcciones mentales; esto es, que los seres humanos aprenden construyendo nuevas ideas o conceptos, en base a conocimientos actuales y previos (Karlin \& Vianni, 2001).

Por lo que en el Aprendizaje Basado en Proyectos se desarrollan actividades de aprendizaje interdisciplinarias, de largo plazo y centradas en el estudiante. (Challenge 2000 Multimedia Project, 1999).

Las características del ABP De Graaf (2007) son las siguientes:

- Los problemas complejos y no estructurados, sacados de la vida real, constituyen el foco y el estímulo del curso, currículum o programa.

- El aprendizaje es centrado en el estudiante

- El profesor adquiere el papel de facilitador

- El aprendizaje se lleva a cabo en pequeños grupos de estudiantes que estudian, analizan, contrastan y proponen soluciones al problema planteado

- La evaluación del aprendizaje es realizada por el propio alumno y por sus compañeros.

Para el diseño de proyectos se requiere lo siguiente: etapa de análisis y planeación del proyecto, se debe formular un objetivo definido, limitación del problema o situación a resolver, identificación de los perfiles de los actores involucrados, desarrollar el proyecto que lleve a resultados y conclusiones de acuerdo a los objetivos planteados.

Trabajo colaborativo: es un proceso intencional de un grupo para alcanzar objetivos específicos. En el marco de una organización, el trabajo en grupo con soporte tecnológico se presenta como un conjunto de estrategias tendientes a maximizar los resultados y minimizar la pérdida de tiempo e información en beneficio de los objetivos organizacionales.

Haciendo mención a los modelos organizativos del futuristas, Tom Peters menciona que...la organización exitosa del futuro tendrá la capacidad para gerenciar la imaginación de las personas, ya que esto es lo que creará el valor de mercado en una corporación ... con ello Peters establece que llegamos a la "Era de la imaginación", es decir que sólo podrán prosperar aquellas personas con verdadera pasión por el aprendizaje y por la adaptación en una organización.

Continúa comentando que..." el nuevo motor del progreso es dividir a las grandes corporaciones en unidades independientes que funcionen como empresas independientes..." por lo que la estructura más efectiva y rentable para la empresa del futuro, es un pequeño núcleo de empleados fijos que protegen las ventajas competitivas de la empresa, y una vasta red de especialistas (o nombres en un rolodex o directorio telefónico) que pueden ser utilizados conforme surjan las necesidades. 
Existen dos modelos que pueden ilustrar lo que será la corporación del futuro:

1.- La organización tipo trébol: sea una empresa como las tres hojas de un trébol. Una de las hojas es un pequeño grupo de empleados permanente, que cuidan la destreza principal de la empresa - su ventaja competitiva. La segunda hoja del trébol la conforman la esencia del trabajo, lo hacen especialistas o contratistas. La tercera hoja son los empleados temporales que hacen el resto del trabajo cotidiano.

2.- La organización tipo estudio de Hollywood: La mayoría de los estudios consisten en un pequeño grupo de empleados permanentes, que tienen una vasta red de contratistas a su disposición. Cuando comienzan a trabajar en una película, este personal puede apalancar su efectividad, trayendo al mejor talento disponible. Al finalizar el proyecto, toda la organización puede desmantelar sin ningún costo adicional; quedando libres para emprender el próximo proyecto, que puede requerir de un equipo totalmente distinto.

Es por lo que se considera que las empresas deben organizarse por proyectos: un objetivo concreto, al que se llega organizando unos determinados recursos para aprovechar una cierta oportunidad que, con algo de suerte, creará un monopolio, eso sí, temporal. Nada de cargas estructurales, excepto las imprescindibles: que cada parte de la cadena de valor haga su labor en la que se es especialista.

De proyecto en proyecto, hasta el próximo desafío. No hay nóminas de estrellas, de directores, de iluminadores: cada uno es su propia empresa, y deben gestionarse como marcas.

Trabajar con equipos de talento organizados temporalmente alrededor de un proyecto, toda la intensidad y conocimiento centrados durante el mismo y acabado el proyecto, disuelto el equipo.

La clave para el éxito es doble: disponer del mapa de expertos para saber localizar los mejores ante un proyecto y ser capaz de ejercer el liderazgo sobre una tribu de personalidades.

De tal forma que la importancia pedagógica de Aprendizaje aplicado a las asignaturas de especialidad en problemas reales; tal como lo hace un médico o una enfermera en campo, lo que los lleva al éxito logrado de Aprendizaje Servicio como estrategia educativa para desarrollar competencias que incrementa la sistematización de esta práctica; como lo demuestra G. Tolsa Jara (2021).

\section{METODOLOGÍA APLICADA}

Esta investigación es de corte cuantitativo; se realizó la recolección de datos con el fin de explicar las causas que implicarán en el alumno de pasar de un aprendizaje basado en proyectos colaborativos hasta llegar a desarrollar un proyecto desde el punto de vista organizacional tipo Hollywood (causaefecto).

El objetivo de la investigación es conocer si posible lograr un enfoque organizacional tipo Hollywood a través del aprendizaje basado en proyectos colaborativos. Por lo que es una investigación 
descriptiva al observar cómo se manifiesta su comportamiento organizacional para desarrollar un proyecto de forma grupal. Dado que se trabajó sobre las realidades de un hecho al conocer las constantes de un fenómeno; por el método empleado en el objetivo es de tipo sintético-deductivo; es decir partiendo de lo universal se llegó a un caso particular al deducir el efecto partiendo de la causa.

POBLACIÓN DEL ESTUDIO. El grupo sobre el cual se trabajó la investigación fue de Octavo Semestre de la Carrera de Ingeniería en Electrónica, es un grupo integrado por 20 alumnos, cuyas edades oscilan entre los 20 y 24 años. Cabe mencionar que los alumnos desde el segundo semestre realizan proyectos de aplicación a una problemática real al final de cada semestre.

Es un grupo en el cual se han conocido durante la carrera y eligen libremente con quien trabajar para desarrollar dichas aplicaciones, las cuales deben englobar las materias que están cursando en determinado semestre para sus evaluaciones respectivas.

DESCRIPCIÓN DE LA EXPERIMENTACIÓN. La presente investigación se realizó durante un semestre para la materia de Control de Procesos; durante las tres evaluaciones parciales los alumnos realizaron un proyecto por equipos; el cual consistió en buscar una problemática a resolver dando aplicación a su carrera y a la materias que cursaban en ese semestre; siguiendo el protocolo de investigación que marca el COMECYT; esto porque algunos proyectos participan en la Feria Mexicana de la Ciencia, así que damos esa formalidad en las investigaciones. En esta primera etapa se consideró al grupo experimental G1.

Al terminar sus proyectos se conjuntó a todo el grupo para seguir con la segunda etapa, por lo que se convirtieron en el grupo experimental G2. Para esta etapa, el docente les propuso de forma grupal una problemática real a resolver; siguiendo el mismo protocolo de investigación del Consejo Mexiquense de la Ciencia y Tecnología (COMECYT).

Para la presente investigación se aplicaron Series Cronológicas, en las cuales se analizó el objeto de una variable en sucesivos intervalos de tiempo. Mediante el análisis de estas series, fue posible determinar la tendencia general del fenómeno. Se realizó una comparación del grupo experimental G1 Vs el grupo experimental G2, realizando las observaciones con sus problemáticas respectivamente.

Donde las problemáticas a considerar fueron:

Problemática 1. Dar solución a una problemática real propuesta por ellos mismos, trabajando en equipos, con lo cual aplican el ABP.

Problemática 2. Dar una solución a una problemática real de forma grupal propuesta por el profesor. Donde se les explicó que esta era una simulación pensada que una empresa les había pedido realizar un 
proyecto para monitorear la seguridad del peatón, la seguridad del automovilista y la seguridad de quien se desplaza en metro; considerando una avenida principal X.

La técnica para la recolección de datos fue por medio de la observación de puntos clave en el comportamiento de los alumnos; tanto para trabajar en grupos pequeños, como de forma grupal en el proyecto integral. Dichos puntos se fueron registrando en una Batería de Pruebas de Habilidades Cognoscitivas (BPHC) generadas de acuerdo con lo expuesto anteriormente y dando un valor a estas con un escalamiento de Likert.

Posteriormente se les explicó que acababan de experimentar una Organización tipo HOLLYWOOD; en donde todos se organizaron para dar una solución a una problemática propuesta. Para evaluar este punto en particular se les aplicó una encuesta a los alumnos con el fin de conocer si ellos veían factible esta modalidad para su situación real al enfrentarse en el campo laboral.

Con la BPHC se evalúan las siguientes habilidades cognoscitivas: análisis, síntesis, inducción, deducción, abstracción y generalización; así como también el nivel de desarrollo en tres tipos de pensamiento: lógico, analítico y estructural. Para caracterizar habilidades cognoscitivas y desarrollo del pensamiento se evalúan además de una serie de funciones psicológicas superiores: percepción, atención y memoria. Por otra parte se evalúan las operaciones cognoscitivas de identificación, distinción, comprensión visual, organización, organización estructural y la solución a la problemática.

A continuación, se describe la estructura de la BPHC empleada utilizando los puntos mencionados por Feuerstein.

\section{PHC1 para G1 y PHC2 para el G2 en ABP}

Contenido: Para las pruebas los alumnos realizan un protocolo de investigación, en la primera evaluación parcial plantean lo siguiente: una problemática real a resolver, antecedentes, justificación, objetivos, hipótesis, marco teórico, metodología para dar una solución al problema, cronograma de actividades y bibliografía. En la segunda evaluación parcial realizan lo anterior registrando resultados y discusiones parciales; así como sus conclusiones parciales. Para la tercera evaluación parcial concluyen con su trabajo escrito final y con el prototipo funcionando de acuerdo a sus objetivos.

Considerar que para la PCH1, los alumnos trabajan en equipos de 3 personas cada uno y para la $\mathrm{PCH} 2$, los alumnos trabajan para una problemática real integral propuesto por el profesor. Siguiendo el mismo rigor metodológico que se ha mencionado apegados al cronograma de actividades propuesto por ellos mismos para cada prueba. 
Un punto importante en esta última prueba es que los alumnos designaron quien sería el líder el proyecto, quienes estarían en la cabeza de las tres etapas a desarrollar y los compañeros que estarían en cada una de las etapas para desarrollar.

Objetivo. Comprobar mediante ABP la aplicación práctica de las habilidades cognoscitivas (análisis, síntesis, inducción, deducción, abstracción y generalización).

\section{Operaciones cognoscitivas:}

Solución de una problemática real, como la forma de manifestar el pensamiento en general.

Comprensión analítica, comprender la problemática y la forma de estructurar el prototipo que resolverá la situación. (recordar, reconocer información específica MEMORIZAR)

> Identificación de procesos abstractos, los pasos a seguir para plantear el modelo y la forma funcional de toda la estructura a construir. (explica, parafrasea COMPRENDER)

$>$ Comprensión dimensional. Consiste en la representación por medio de diagramas para la construcción del proyecto. (resuelve, determina, APLICAR)

$>$ Comprensión estructural. En este momento ya se cuenta con suficientes elementos para comenzar la construcción del proyecto físicamente. (clasifica, modela, interpreta ANALIZA)

Exploración de la capacidad de concentración.

Asimilación. Si el alumno está en la posibilidad correcta de lograr el objetivo del proyecto. (juzga, selecciona, critica, justifica EVALUA)

> Generalización, Es posible que el alumno pueda seguir aplicando esta forma didáctica. (propone, inventa, crea, diseña, mejora CREAR)

Funciones psicológicas predominantes. Concentración y memoria.

Modalidad. Simbólica y Práctica.

Tipo de Pensamiento. Lógico y Crítico

Habilidad cognoscitiva Generalización

Nivel de concreción. Es alta porque requiere de una gran habilidad cognoscitiva y de concentración.

Instrucciones. Se explican al inicio del semestre.

Las baterías quedaron conformadas considerando 8 puntos con sus respectivos aspectos a evaluar; a los cuales se les asignó una puntuación de acuerdo a la escala de Likert. 
MUY BUENO

BUENO

REGULAR

DEFICIENTE

Con respecto a la aplicación piloto y ajuste de instrumento, se realizó una prueba de observación previa, con el fin de detectar si los puntos que se presentaban eran suficientes para poder obtener un resultado y observar si el tipo de proyecto era el adecuado de acuerdo a sus conocimientos teóricos y en la lógica, para poder identificar las habilidades que se deseaban estudiar.

Para la evaluación con respecto al modelo estilo Hollywood se analizaron 8 preguntas a las que les fueron fijadas las siguientes puntuaciones de acuerdo a la escala de Likert.

EXCELENTE

ACEPTABLE

POCO ACEPTABLE (1)

DEFICIENTE

(0)

\section{ANÁLISIS DE RESULTADOS}

A continuación se muestra la Tabla 1 con los valores obtenidos en la primera observación con el grupo G1 ABP en trabajo por equipos, así como su gráfica ante estos resultados. (ver Gráfica 1). La figura 2 muestra el analisis estadistico del comportamiento del estudio y los avances obtenidos, se observa principalmente la ecuación característica y el alcance obtenido con la prueba. 
TABLA 1. Resultados del grupo G1

\begin{tabular}{|l|c|c|c|c|c|c|c|c|}
\hline \multicolumn{1}{|c|}{ ALUMNOS } & $\mathrm{A}$ & $\mathrm{B}$ & $\mathrm{C}$ & $\mathrm{D}$ & $\mathrm{E}$ & $\mathrm{F}$ & $\mathrm{G}$ & $\mathrm{H}$ \\
\hline AMADO MAURO URIEL & 3 & 3 & 2 & 3 & 3 & 2 & 3 & 3 \\
\hline ARROYO GARCİA CARLOS & 3 & 3 & 3 & 3 & 3 & 3 & 3 & 3 \\
\hline BAUTISTA BAUTISTA EDGAR ALAN & 3 & 3 & 3 & 3 & 2 & 3 & 3 & 3 \\
\hline BENITES GUTIERREZ EMMANUEL & 3 & 3 & 3 & 3 & 3 & 3 & 3 & 3 \\
\hline CASIMIRO GONZÀLEZ CESAR & 2 & 2 & 2 & 2 & 2 & 2 & 3 & 2 \\
\hline CHAVEZ PEREZ MARIANA & 3 & 2 & 2 & 3 & 2 & 2 & 2 & 2 \\
\hline CUEVAS CERVANTES TANIA & 3 & 3 & 2 & 3 & 3 & 3 & 3 & 2 \\
\hline DELGADO FLORES VICTOR MANUEL & 3 & 3 & 3 & 3 & 2 & 3 & 3 & 3 \\
\hline FLORES ESCOBAR ISRAEL & 3 & 2 & 3 & 2 & 2 & 2 & 2 & 2 \\
\hline FRANCISCO PALACIOS DAVID & 2 & 2 & 2 & 3 & 2 & 2 & 2 & 3 \\
\hline GUZMÁN GOMEZ VLADIMIR & 3 & 3 & 3 & 3 & 2 & 3 & 3 & 3 \\
\hline HERNÀNDEZ SUAREZ ERIKA & 3 & 3 & 3 & 3 & 2 & 3 & 3 & 3 \\
\hline MEDINA OSORIO SANDRA & 2 & 2 & 2 & 2 & 2 & 2 & 2 & 2 \\
\hline MONTES RAMIREZ PAVEL & 3 & 3 & 3 & 3 & 2 & 3 & 3 & 3 \\
\hline MORENO CARDENAS JOSĖ & 2 & 2 & 2 & 3 & 2 & 2 & 2 & 2 \\
\hline PEREZ BARRAGAN ROBERTO & 3 & 2 & 3 & 2 & 3 & 2 & 2 & 2 \\
\hline PULIDO RUEBA FERNANDO & 2 & 3 & 2 & 3 & 2 & 2 & 2 & 2 \\
\hline RESENDIZ MARTINEZ & 3 & 2 & 2 & 2 & 2 & 2 & 2 & 2 \\
\hline RICO TORRES LUIS EDUARDO & 2 & 2 & 2 & 3 & 2 & 2 & 2 & 2 \\
\hline ZAMUDIO CARBAJAL NERY ARTURO & 3 & 3 & 3 & 3 & 3 & 3 & 3 & 3 \\
\hline PROMEDIOS & $\mathbf{2 . 7}$ & $\mathbf{2 . 5 5}$ & $\mathbf{2 . 5}$ & $\mathbf{2 . 7 5}$ & $\mathbf{2 . 3}$ & $\mathbf{2 . 4 5}$ & $\mathbf{2 . 5 5}$ & $\mathbf{2 . 5}$ \\
\hline
\end{tabular}

GRAFICA 1. Resultados gráficos del grupo G1

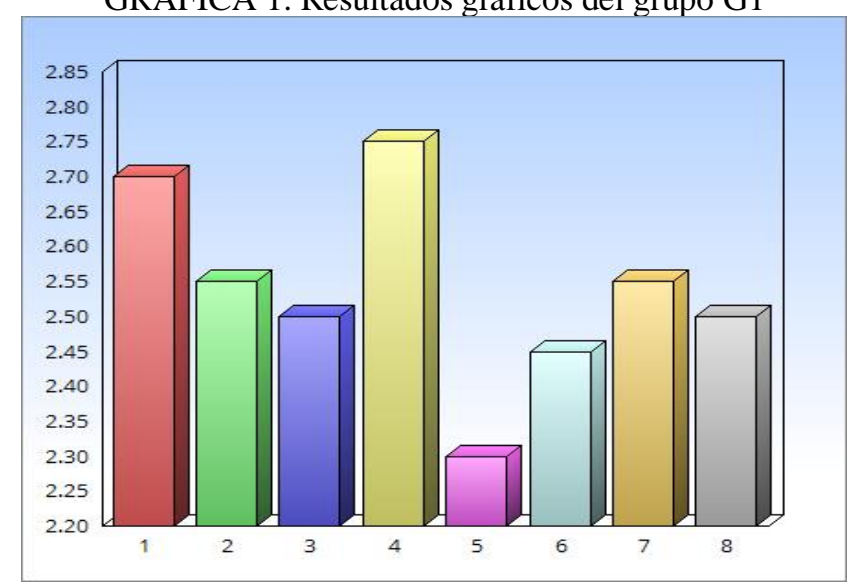


Fig. 2. Analisis estadistico del comportamiento.

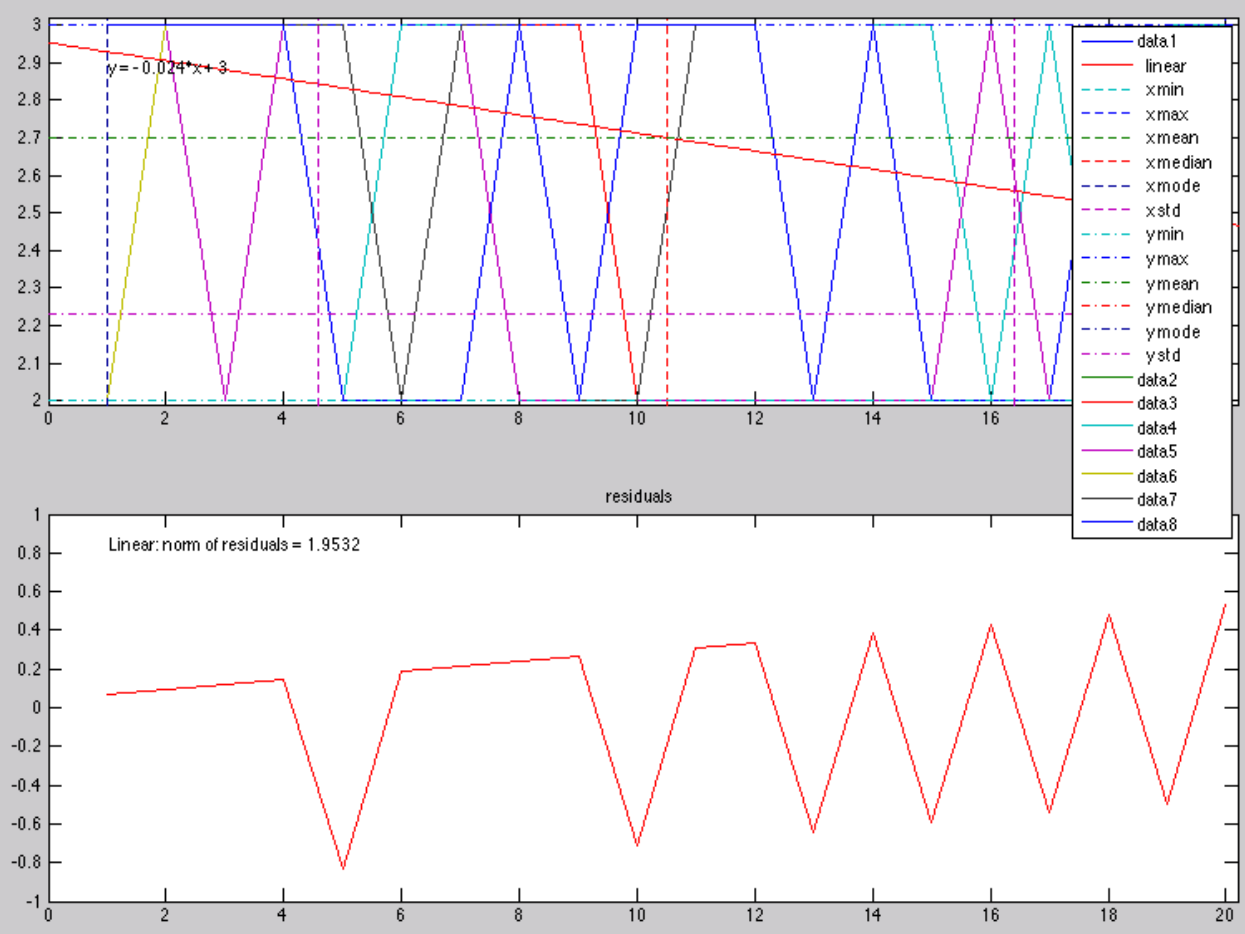

A continuación se presenta la Tabla 2 de resultados con su respectiva Gráfica 2. De la segunda observación con el grupo G2 ABP, considerando la problemática propuesta por el profesor en forma grupal. La figura 3 muestra el analisis estadistico del comportamiento del estudio y los avances obtenidos, se observa principalmente la ecuación característica y el alcance obtenido con la prueba.

TABLA 2. Resultados del grupo G2

\begin{tabular}{|l|c|c|c|c|c|c|c|c|}
\hline \multicolumn{1}{|c|}{ ALUMNOS } & $\mathrm{A}$ & $\mathrm{B}$ & $\mathrm{C}$ & $\mathrm{D}$ & $\mathrm{E}$ & $\mathrm{F}$ & $\mathrm{G}$ & $\mathrm{H}$ \\
\hline AMADO MAURO URIEL & 2 & 3 & 3 & 3 & 3 & 3 & 3 & 3 \\
\hline ARROYO GARCİA CARLOS & 3 & 3 & 3 & 3 & 3 & 3 & 3 & 3 \\
\hline BAUTISTA BAUTISTA EDGAR & 2 & 3 & 3 & 3 & 3 & 3 & 3 & 3 \\
\hline BENITEZ GUTIERREZ EMMANUEL & 3 & 3 & 3 & 2 & 3 & 3 & 2 & 2 \\
\hline CASIMIRO GONZÀLEZ CESAR & 2 & 3 & 3 & 3 & 3 & 3 & 3 & 3 \\
\hline CHAVEZ PEREZ MARIANA & 2 & 3 & 2 & 2 & 3 & 3 & 1 & 1 \\
\hline CUEVAS CERVANTES TANIA & 3 & 3 & 3 & 2 & 3 & 2 & 1 & 1 \\
\hline DELGADO FLORES VICTOR M. & 3 & 3 & 3 & 2 & 3 & 3 & 3 & 3 \\
\hline FLORES ESCOBAR ISRAEL & 2 & 3 & 3 & 2 & 3 & 3 & 3 & 3 \\
\hline FRANCISCO PALACIOS DAVID & 2 & 2 & 2 & 2 & 2 & 2 & 2 & 1 \\
\hline GUZMÁN GOMEZ VLADIMIR & 2 & 3 & 3 & 2 & 3 & 3 & 3 & 3 \\
\hline HERNÀNDEZ SUAREZ ERIKA & 2 & 3 & 3 & 2 & 2 & 3 & 3 & 3 \\
\hline MEDINA OSORIO SANDRA & 3 & 3 & 3 & 2 & 3 & 3 & 2 & 0 \\
\hline MONTES RAMIREZ PAVEL & 3 & 3 & 3 & 2 & 3 & 3 & 3 & 3 \\
\hline MORENO CARDENAS JOSĖ & 3 & 3 & 3 & 2 & 3 & 3 & 3 & 2 \\
\hline PEREZ BARRAGAN ROBERTO & 3 & 2 & 2 & 2 & 2 & 3 & 3 & 3 \\
\hline PULIDO RUEBA FERNANDO & 3 & 3 & 3 & 2 & 3 & 3 & 3 & 3 \\
\hline RESENDIZ MARTINEZ & 2 & 3 & 2 & 2 & 3 & 3 & 3 & 2 \\
\hline RICO TORRES LUIS EDUARDO & 2 & 2 & 3 & 3 & 3 & 3 & 3 & 2 \\
\hline ZAMUDIO CARBAJAL NERY A. & 1 & 3 & 3 & 3 & 3 & 3 & 2 & 3 \\
\hline PROMEDIOS & $\mathbf{2 . 4}$ & $\mathbf{2 . 8 5}$ & $\mathbf{2 . 8}$ & $\mathbf{2 . 3}$ & $\mathbf{2 . 8 5}$ & $\mathbf{2 . 9}$ & $\mathbf{2 . 6}$ & $\mathbf{2 . 3 5}$ \\
\hline
\end{tabular}


GRAFICA 2. Resultados gráficos del grupo G2

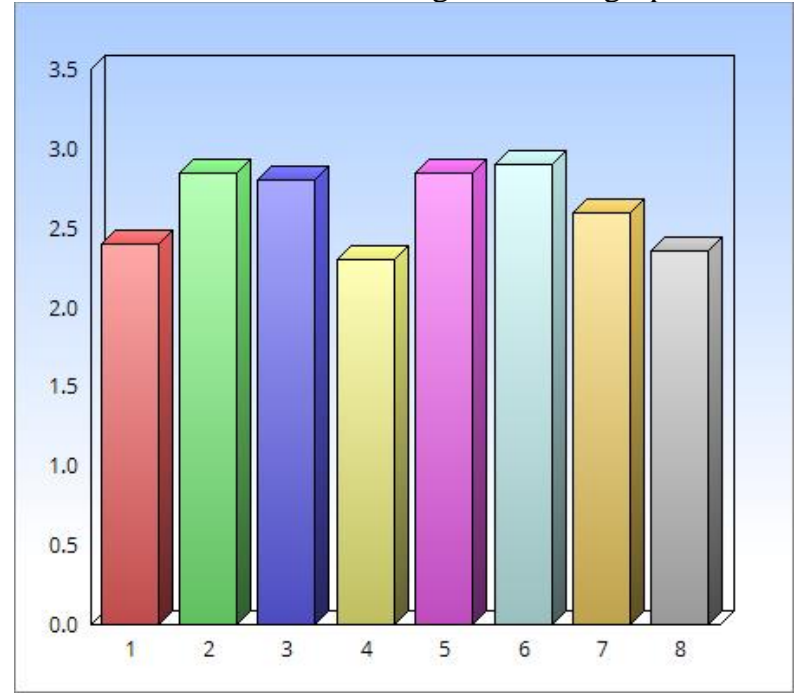

Fig. 3. Analisis estadistico del comportamiento.
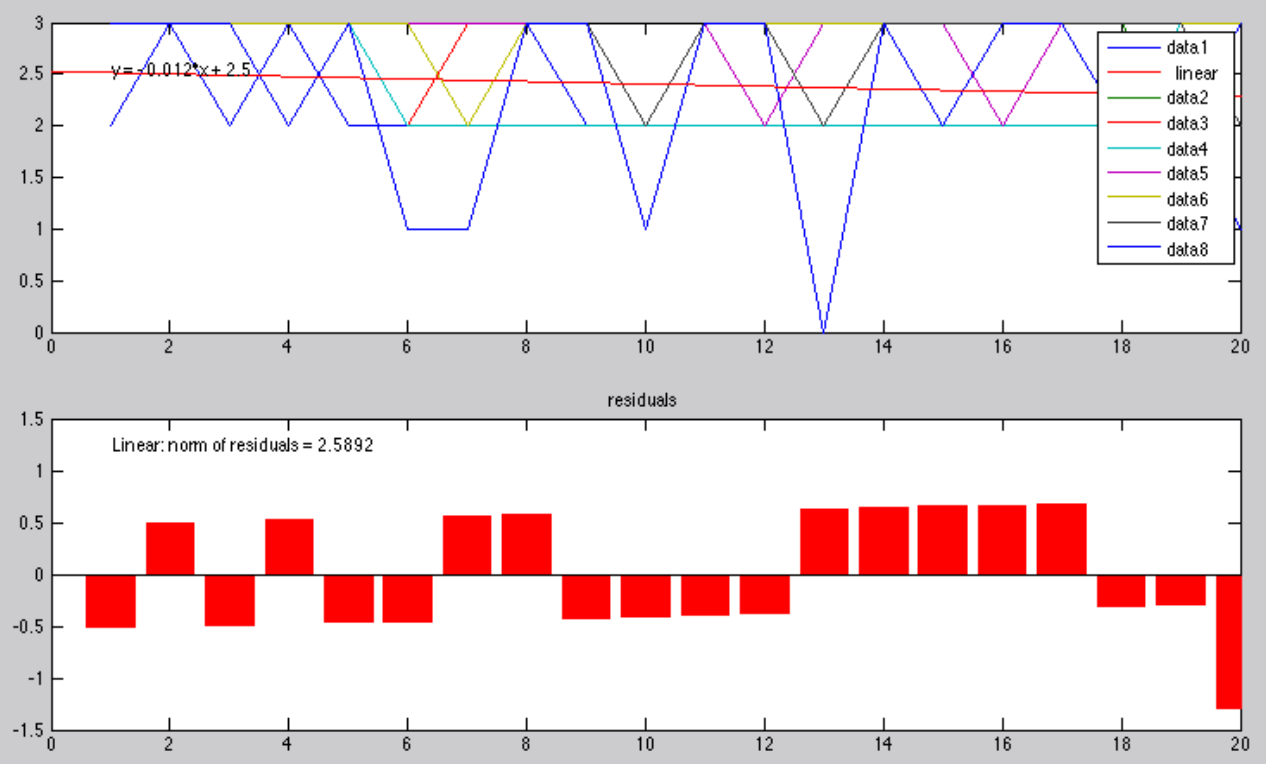

Haciendo una comparación entre ambas formas de trabajar se observó lo siguiente:

1. Para dar una solución a la problemática se observa que se ponen más rápido de acuerdo en grupos pequeños.

2. Su comprensión analítica se vio mejorada al trabajar en forma grupal

3. Al realizar la secuencia que los llevaría a la solución de cada etapa mejoraron en forma grupal.

4. Mas sin embargo cuando unieron esas etapas le costó un poco de trabajo visualizar si sería la mejor solución de forma grupal, se llevaron más tiempo de análisis. 
5. Su comprensión estructural mejoró en forma grupal.

6. Su concentración mejoró en forma grupal, se observó que disfrutaban la etapa en donde sus compañeros los designaron.

7. Su asimilación para lograr el objetivo se manifiesta muy aproximada en ambos casos.

8. Al conjuntar todo el proyecto, hay algunas fallas muy mínimas, tienen menos fallas en forma de equipos.

Para el análisis del modelo estilo Hollywood se muestran los resultados obtenidos en la encuesta planteada a los alumnos, ver Tabla 3 y Gráfica 3. La figura 4 muestra el analisis estadistico del comportamiento del estudio y los avances obtenidos, se observa principalmente la ecuación característica y el alcance obtenido con la prueba.

TABLA 1. Resultados del modelo estilo Hollywood

\begin{tabular}{|c|c|c|c|c|c|c|c|c|}
\hline ALUMNOS & $\mathrm{A}$ & $\mathrm{B}$ & $\mathrm{C}$ & $\mathrm{D}$ & $\mathrm{E}$ & $\mathrm{F}$ & $\mathrm{G}$ & $\mathrm{H}$ \\
\hline AMADO MAURO URIEL & 2 & 3 & 3 & 3 & 3 & 3 & 3 & 3 \\
\hline ARROYO GARCÌA CARLOS & 3 & 3 & 3 & 3 & 3 & 3 & 3 & 3 \\
\hline BAUTISTA BAUTISTA EDGAR ALAN & 2 & 3 & 3 & 3 & 3 & 3 & 3 & 3 \\
\hline BENITES GUTIERREZ EMMANUEL * & 3 & 3 & 3 & 2 & 3 & 3 & 2 & 2 \\
\hline CASIMIRO GONZÀLEZ CESAR & 2 & 3 & 3 & 3 & 3 & 3 & 3 & 3 \\
\hline CHAVEZ PEREZ MARIANA & 2 & 3 & 2 & 2 & 3 & 3 & 1 & 1 \\
\hline CUEVAS CERVANTES TANIA & 3 & 3 & 3 & 2 & 3 & 2 & 1 & 1 \\
\hline DELGADO FLORES VICTOR MANUEL & 3 & 3 & 3 & 2 & 3 & 3 & 3 & 3 \\
\hline FLORES ESCOBAR ISRAEL & 2 & 3 & 3 & 2 & 3 & 3 & 3 & 3 \\
\hline FRANCISCO PALACIOS DAVID & 2 & 2 & 2 & 2 & 2 & 2 & 2 & 1 \\
\hline GUZMÁN GOMEZ VLADIMIR & 2 & 3 & 3 & 2 & 3 & 3 & 3 & 3 \\
\hline HERNÀNDEZ SUAREZ ERIKA & 2 & 3 & 3 & 2 & 2 & 3 & 3 & 3 \\
\hline MEDINA OSORIO SANDRA & 3 & 3 & 3 & 2 & 3 & 3 & 2 & 0 \\
\hline MONTES RAMIREZ PAVEL & 3 & 3 & 3 & 2 & 3 & 3 & 3 & 3 \\
\hline MORENO CARDENAS JOSĖ & 3 & 3 & 3 & 2 & 3 & 3 & 3 & 2 \\
\hline PEREZ BARRAGAN ROBERTO & 3 & 2 & 2 & 2 & 2 & 3 & 3 & 3 \\
\hline PULIDO RUEBA FERNANDO & 3 & 3 & 3 & 2 & 3 & 3 & 3 & 3 \\
\hline RESENDIZ MARTINEZ & 2 & 3 & 2 & 2 & 3 & 3 & 3 & 2 \\
\hline RICO TORRES LUIS EDUARDO & 2 & 2 & 3 & 3 & 3 & 3 & 3 & 2 \\
\hline ZAMUDIO CARBAJAL NERY & 1 & 3 & 3 & 3 & 3 & 3 & 2 & 3 \\
\hline PROMEDIOS & 2.4 & 2.85 & 2.8 & 2.3 & 2.85 & 2.9 & 2.6 & 2.35 \\
\hline
\end{tabular}

GRÀFICA 3. Resultados del modelo estilo Hollywood

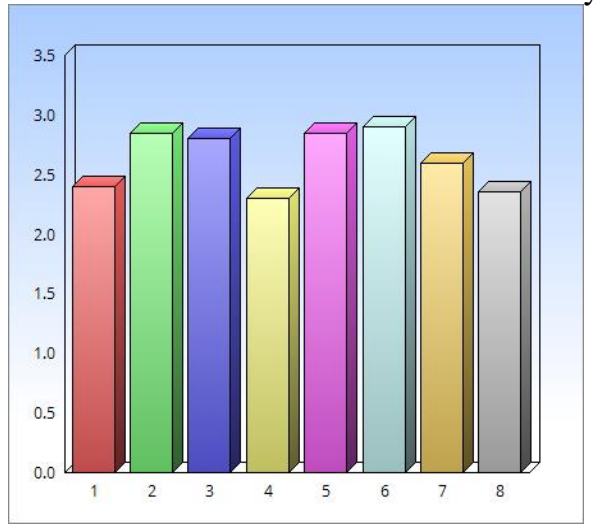


Fig. 4. Analisis estadistico del comportamiento.
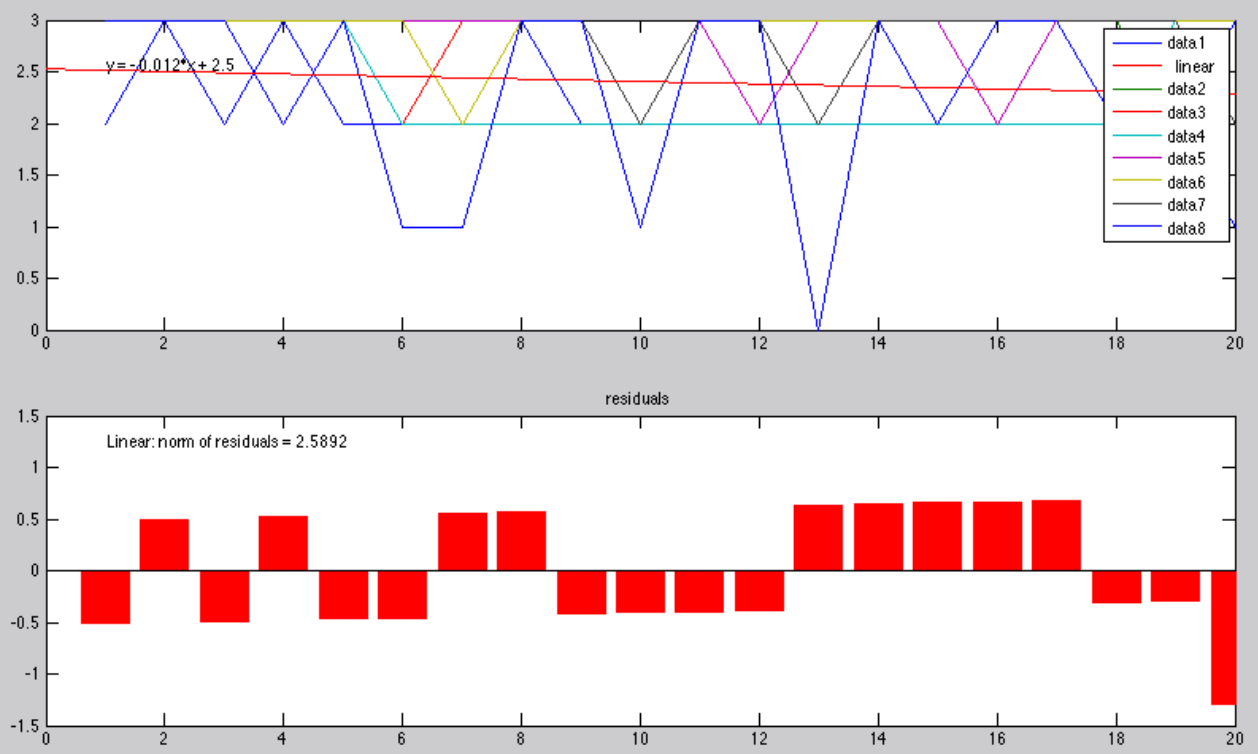

\section{CONCLUSIONES}

En general siguieron mostrando el mismo interés con la dinámica grupal y con la dinámica como han trabajado en el ABP; el organizarse de forma grupal les costó un poco más de trabajo; pero hasta que uno de los alumnos se paró al frente para dimensionar el proyecto, estableciendo las etapas principales y revisando el objetivo del proyecto; tal y como lo han realizado de forma protocolaria.

Posteriormente eligieron un líder; el cual fue el jefe de grupo, quien ha sido jefe de grupo por 5 semestres consecutivos, eso me llamó la atención; implica que en efecto a desempeñado un buen papel. Continuaron con la elección de los 3 representantes para cada etapa; se observó con los resultados obtenidos para esta fase, que efecto fueron las personas correctas. Finalmente, los electos para cada etapa eligieron con el apoyo del grupo a las personas que trabajarían con ellos.

Se observa que todos los alumnos saben cuáles son las habilidades, cualidades y fortalezas de sus compañeros para aprovecharlas al máximo; mas sin embargo al preguntarles si ellos mismos estuvieron de acuerdo en el puesto asignado un $70 \%$ considera haberse puesto en otra etapa diferente. Esto no fue obstáculo para no trabajar, el grupo consideró y aprovecho al máximo o aprendido en los semestres con el ABP.

Al preguntarles que les pareció el trabajar simulando el modelo organizacional tipo Hollywood; se manifiesta que un $86 \%$ le pareció una buena opción, se sintieron a gusto; más sin embargo al considerar esa idea en un momento dado; un $78 \%$ podría considerarla; se sienten preparados para desempeñarse en esta modalidad y el $22 \%$ restante manifestó que quisiera tener un sueldo fijo y eso les causaría cierta inseguridad económica. 
Por lo que se concluye finalmente que se sienten preparados para desempeñarse en esta modalidad; que el aprendizaje basado en proyectos colaborativos si propicia a trabajar en la modalidad organizacional tipo Hollywood y también considerando que han trabajado semestre con semestre proyectos diferentes estarían dispuestos a realizarlos para problemáticas reales. 


\section{BIBLIOGRAFÌA}

[1] Díaz, B. F (2006) Concepción actual de la estrategia de proyectos y competencias que promueve. En Enseñanza situada. Vínculo entre la escuela y la vida. México Mc Graw Hill..

[2] UFAP (...) Aprendizaje basado en problemas. Disponible en: http://ufap.dgdp.uaa.mx/descargas/abp_aprendizaje.pdf Consultado: Abril 8, 2015

[3] Díaz, B. F (2006) La importancia del rol del docente como tutor en el ABP. En Enseñanza situada. Vínculo entre la escuela y la vida. México. Mc Graw Hill.

[4] Peters, T. (2003). El seminario de Tom Peters. Los tiempos de locura requieren de empresas locas. Argentina: Ed. Atlántida

[5] Seminario de Tom Peters. Disponible en: https://prezi.com/rbxj1m3hb1fq/copy-of-copy-of-copy-ofseminario-de-tom-peters/ Consultado: Junio 10, 2015

[6] Ingeniería en Gestión Empresarial, Diseño Organizacional. Dra. Patricia Rivera Acosta. Disponible en: http://es.slideshare.net/terenavarro585/estructuras-tipos Consultado: Julio 13, 2015

[7] RESTREPO-GÓMEZ, B. Aprendizaje basado en problemas (ABP): una innovación didáctica para la enseñanza universitaria. Educación y Educadores, 2005, 8, 9-19.

[8] Valdez Alemán, Eva; Medina Santiago, Alejandro; García de Luna, Beatríz. Uso de Software educativo en la solución de problemas. Epistme, 2006, no. 7. Año 2, enero-marzo

[9] Bermudes Aponte, José J.; Medrano León, Elvia C. La Resolución de problemas a través de Estudio de Casos; una experiencia en estudiantes y profesores de pedagogía infantil de la universidad de la Sabana. Revista educación y desarrollo Social 1: 81-94, 2011

[10] COMO LOGRAR ORGANIZACIONES MODERNAS Y EXITOSAS; Agosto 8, 2014. Por: Dr. Eduardo Chavarri Joo Director de la Escuela de Posgrado de la UNIDAMÁS ALLA DEL CAMBIO: hacia el abandono de todo. Disponible en: http://www.unida.edu.py/blog/2014/08/08/como-lograrorganizaciones-modernas-y-exitosas/ Consultado: Julio 15, 2015

[11] RODRÍGUEZ-SANDOVAL, E.; VARGAS-SOLANO, E.; ESPEJO, C.; ESTRADA-KASSIR, E.; LOZANO, A.; CONTENTO, R. Estrategia pedagógica para promover la continuidad del aprendizaje en la formación en ingeniería. XXVIII Reunión Nacional de Facultades de Ingeniería y VI Encuentro Iberoamericano de Instituciones de Enseñanza de la Ingeniería. "El profesor de ingeniería, profesional de la formación de ingenieros". Asociación Colombiana de Facultades de Ingeniería (Acofi). Cartagena de Indias, 2007, p. 55.

[12] ALPTEKIN, SE.; DETURRIS, D.; MACY, DJ. Development of a flying eye: A project-based learning experience. Journal of Manufacturing Systems, 2005, Vol. 24, No. 3, p. 226-236.

[13] ARAZ, G., \& SUNGUR, S. The interplay between cognitive and motivational variables in a problembased learning environment. Learning and Individual Differences, 2007, 17, 291-297. 
[14] BECERRA-LABRA, C.; GRAS-MARTÍ, A.; MARTÍNEZ-TORREGROSA, J. La física con una estructurada problematizada: efectos sobre el aprendizaje conceptual, las actitudes e intereses de los estudiantes universitarios. Revista Brasileira de Ensino de Física, 2007, 29 (1), 95-103.

[15] FALLIK, O.; EYLON, BS.; ROSENFELD, S. Motivating teachers to enact free-choice project-based learning in science and technology (PBLSAT): Effects of a professional development model. Journal of Science Teacher Education, 2008, 19, 565-591.

[16] LIU, WC.; WANG, CKJ.; TAN, OS.; KOH, C.; EE, J. A self-determination approach to understanding students' motivation in project work. Learning and Individual Differences, 2008. DOI:10.1016/j.lindif.2008.07.002.

[17] MARTÍNEZ-RODRIGO, F.; HERRERO DE LUCAS, LC.; GONZÁLEZ DE LA FUENTE, JM.; DOMÍNGUEZ-VÁZQUEZ, JA. Project based learning experience in industrial electronics and industrial applications design. Universidad de Valladolid. Escuela Universitaria Politécnica, 2007. [Consultado 2015.] En:http://www.greidi.uva.es/articulos/EUP_ProjectBased.pdf.

[18] METTAS, AC.; Constantinou, CC. The technology fair: a project-based learning approach for enhancing problem solving skills and interest in design and technology education. International Journal of Technology and Design Education, 2007, 18, 79-100.

[19] MILENTIJEVIC, I.; CIRIC, V.; VOJINOVIC, O. Version control in project-based learning. International Computers \& Education, 2008, 50, 1331-1338.

[20] MIODUSER, D.; BETZER, N. The contribution of Project-based-learning to high-achievers' acquisition of technological knowledge. International Journal of Technology and Design Education, 2007, $18,59-77$.

[21] MOESBY, E.; JOHANNSEN, HHW.; KORNOV, L. Individual activities as an integrated part of project work: an innovative approach to project oriented and problem-based learning (POPBL). World Transactions on Engineering and Technology Education, 2006, 5 (1), 11-17.

[22] VAN DEN BERGH, V.; MORTERMANS, D.; SPOOREN, P.; VAN PETEGEM, P.; GIJBELS, D.; VANTHOURNOUT, G. New assessment modes within project-based education the stakeholders. Studies in Educational Evaluation, 2006, 32, 345-368.

[23] WATSON, G. Using technology to promote success in PBL courses. The technology source. University of Noth Carolina, May/June 2002. Disponible en : http://technologysource.org/article/using_technology_to_promote_success_in_pbl_courses/. Consultado en 2015
[24]
EDUTEKA
(2006)
Aprendizaje
por
proyectos

http://www.eduteka.org/AprendizajePorProyectos.php Consultado 09/05/15

[25] Tolsa Jara, Gilda. Experiencia: incorporación de aprendizaje servicio en asignaturas de la carrera de enfermería Universidad Santo Tomas. Santiago, Chile. 2017. South Florida Journal of Development. Miami. v.2.n.1. p. 559-565, jan/feb. 2021. ISSIN 2675-5459 